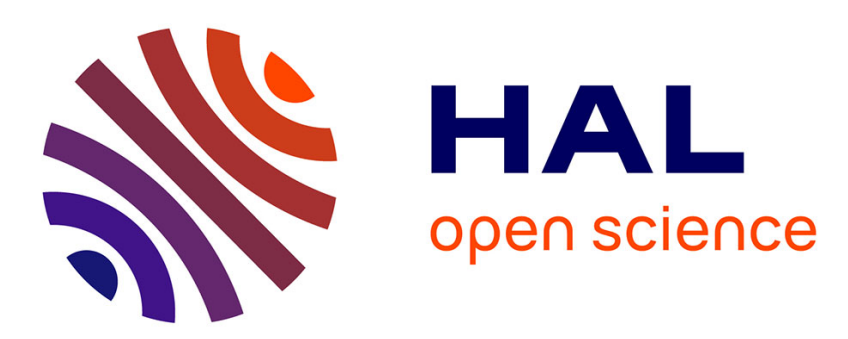

\title{
A picosecond Josephson junction model for circuit simulation
}

\author{
A. de Lustrac, P. Crozat, R. Adde
}

\section{To cite this version:}

A. de Lustrac, P. Crozat, R. Adde. A picosecond Josephson junction model for circuit simulation. Revue de Physique Appliquée, 1986, 21 (5), pp.319-326. 10.1051/rphysap:01986002105031900 . jpa00245449

\section{HAL Id: jpa-00245449 https://hal.science/jpa-00245449}

Submitted on 1 Jan 1986

HAL is a multi-disciplinary open access archive for the deposit and dissemination of scientific research documents, whether they are published or not. The documents may come from teaching and research institutions in France or abroad, or from public or private research centers.
L'archive ouverte pluridisciplinaire HAL, est destinée au dépôt et à la diffusion de documents scientifiques de niveau recherche, publiés ou non, émanant des établissements d'enseignement et de recherche français ou étrangers, des laboratoires publics ou privés. 


\title{
A picosecond Josephson junction model for circuit simulation
}

\author{
A. De Lustrac, P. Crozat and R. Adde \\ Institut d'Electronique Fondamentale, Bâtiment 220, Université Paris-XI, 91405 Orsay, France
}

(Reçu le 9 septembre 1985, révisé le 7 février 1986, accepté le 11 février 1986)

\begin{abstract}
Résumé. - La résolution au premier ordre de l'équation Josephson dépendant du temps permet d'étudier avec précision la dynamique de la commutation des jonctions Josephson de temps de commutation $\simeq 1$ ps. La modélisation obtenue pour la jonction est presque aussi facile d'emploi que le modèle RSJC dans les calculs numériques. Des exemples illustrent le domaine d'application de la méthode par rapport au modèle RSJC en fonction de l'environnement de la jonction.
\end{abstract}

\begin{abstract}
The first order series expansion of the time dependent Josephson equation allows a precise simulation of the switching dynamic of picosecond Josephson junctions. The junction model is quite as efficient as the RSJC model in computer calculations. Numerical examples illustrate its domain of application for picosecond junctions in a circuit environment by comparison with the RSJC model.
\end{abstract}

\section{Introduction.}

The dynamic simulation of Josephson tunnel devices uses extensively the model of the resistively shunted junction with capacitance or $\operatorname{RSJC}$ model [1, 2]. This model is simple and gives fast computations with standard circuit simulators (SPICE, ASTEC). It allows usually an efficient modelling of Josephson tunnel junctions in complex circuits with a satisfactory accuracy. However several works reviewed in [3] point out the model limitations. They show that it becomes less accurate when the time constant $R_{\mathrm{N}} C$ of the Josephson junction reaches the picosecond range. This occurs e.g. in small area planar lead junctions $\left(\sim 1 \mu \mathrm{m}^{2}\right)$ or niobium high current density $\left(\geqslant 5 \times 10^{3} \mathrm{~A} / \mathrm{cm}^{2}\right)$ edge-grown junctions. Relatively simple circuits such as a Josephson sampler [4] have been realized with the latter. The recent advances in the III-V semi-conductor technologies indicate that a competitive Josephson technology must operate at very high speed (picosecond junctions) and allow large integration such as in direct coupled circuits [5]. The precise simulation of such fast Josephson circuits requires a junction model taking into account with a reasonable accuracy the characteristic features of the ac Josephson effect at the frequency components in the range or beyond the gap frequency. On the other side this improvement in accuracy relatively to the RSJC model must not be at too large a cost in terms of computer time and memory space. For- mulations of the full Josephson time dependent equation or Werthamer equation have been given in $[6,7]$. Numerical calculations have been made mainly at the scale of the single device [8-11]. They could be extended hardly to logic gates and even less to more complex circuits.

Starting from a suggestion by Larkin and Ovchinikov [7] we have developed an extension of the RSJC model which is a first order series expansion of the time dependent Josephson equation [11]. It is well suited to tunnel junctions with time constant down to $R_{\mathrm{N}} C \sim 1 \mathrm{ps}$ and constitutes an efficient and useful tool for the simulation of full circuits. We present in this paper an account of the method. We discuss its range of validity between the RSJC model and the full resolution of the Werthamer equation. We give numerical examples illustrating typical situations in circuits.

\section{Limitations of the adiabatic approximation and the RSJC model.}

The adiabatic approximation comes out from the expression of the time dependent Josephson current $I(t)$ in a superconducting tunnel junction as expressed for example in [7, 13]. We reproduce equation (8) of [7] in a slightly modified form which underlines the formulation of the integral versus time of the voltage $V\left(t^{\prime}\right)$ accross the junction. 


$$
\begin{aligned}
& I(t)=\frac{1}{2 \pi^{3} R} \mathcal{R e} \int_{-\infty}^{0} i \mathrm{~d} t_{1}\left\{\iint_{-\infty}^{+\infty} \mathrm{d} \omega_{1} \mathrm{~d} \omega_{2} \exp \left[-i \int_{-\infty}^{t} \frac{e}{\hbar} V\left(t^{\prime}\right) \mathrm{d} t-i \int_{-\infty}^{t+t_{1}} \frac{e}{\hbar} V\left(t^{\prime}\right) \mathrm{d} t^{\prime}\right] \times\right. \\
& \times \exp \left[-i\left(\omega_{1}+\omega_{2}\right) t_{1}\right] \times \operatorname{Jm}\left(F_{1}^{+}\left(\omega_{1}\right) \times F_{2}\left(\omega_{2}\right)\right)-\iint_{-\infty}^{+\infty} \mathrm{d} \omega_{1} \mathrm{~d} \omega_{2} \exp \left[i \int_{t}^{t+t_{1}} \frac{e}{\hbar} V\left(t^{\prime}\right) \mathrm{d} t^{\prime}\right] \\
& \times \exp \left[-i\left(\omega_{1}-\omega_{2}\right) t_{1}\right] \times \operatorname{Jm}\left(G_{1}\left(\omega_{1}\right) \times G_{2}\left(\omega_{2}\right)\right) .
\end{aligned}
$$

The symbols are defined similarly as in [7] : $R_{\mathrm{N}}$ is the junction resistance per unit area in the normal state and $G_{1,2}$ and $F_{1,2}$ are the Green functions for superconductors separated by a potential barrier when no external field is applied. We have adopted the sign conventions discussed in detail by Harris [8] for the different Josephson current components so that the real and imaginary parts of the Josephson current in equation (1) are related by the Kramers-Kronig relations.

Simplifications occur in equation (1) for slowly varying electrical quantities $I(t), V(t)$ with characteristic frequencies small compared to the energy gap frequency of the superconductor $\left(2 \Delta_{0}\right)$. It was pointed out in $[7,10,13]$ that typical values of $t_{1}$ satisfy $t_{1} \simeq \hbar / \Delta \lesssim 0.5$ ps with large gap superconductors. Then the voltage $V(t)$ does not vary on this time scale and in equation (1)

$$
\exp \left[i \int_{t}^{t+t_{1}} \frac{e}{\hbar} V\left(t^{\prime}\right) \mathrm{d} t^{\prime}\right] \simeq \exp \left[i \frac{e}{\hbar} V(t) \times t_{1}\right]
$$

Finally (Eq. (1)) may be written

$$
I(t)=I_{\mathrm{c}}(V) \sin [\varphi(t)]+I_{\mathrm{pqp}}(V) \cos [\varphi(t)]+I_{\mathrm{qp}}(V) .
$$

This is the expression of the Josephson current in the adiabatic (0th order) approximation which includes three voltage dependent current components, the quasiparticle component $I_{\mathrm{qp}}(V)$, the $\sin \varphi$ component $I_{\mathrm{c}}(V)$ and the pair-quasiparticle interference term $I_{\text {pqp }}(V)$. A component $C(\mathrm{~d} V / \mathrm{d} t)$ must be added to (Eq. (3)) to take into account the static capacitance of the device [3].

At low temperatures relatively to $T_{\mathrm{c}}$, and voltages smaller than the gap voltage, $I_{c}(V) \simeq I_{c}(0)$ and $I_{\mathrm{pqp}}(V) \simeq 0$ [3]. The total current in a Josephson junction reduces to

$$
I(t)=I_{\mathrm{c}} \sin [\varphi(t)]+I_{\mathrm{qp}}(V)+C \times \mathrm{d} V / \mathrm{d} t
$$

which is the RSJC approximation.

Let us now comment on the validity of these two models. The adiabatic approximation is valid only if $V(t)$ does not vary, or varies by a very small amount compared to $V_{\mathrm{g}}=(2 \Delta / e)$ on a picosecond scale.
This is well verified in junctions with sufficiently large time constant $R_{\mathrm{N}} C>10$ ps (Mc Cumber parameter $\left.\beta=2 e / \hbar I_{\mathrm{c}} R_{\mathrm{N}}^{2} C \# \pi \Delta / \hbar .\left(R_{\mathrm{N}} C\right) \geqslant 50\right)$. Then very fast voltage variations involving frequencies at the superconducting gap frequency or larger are strongly damped by the junction $R_{\mathrm{N}} C$ time constant, and are limited to a few per cent of the gap voltage on a picosecond scale. These devices have rise times $\geqslant 10 \mathrm{ps}$ and the corresponding logic circuits have typical switching time $\gtrsim 20-30$ ps.

On the other side devices with $R_{\mathrm{N}} C$ product in the 1 ps range have an intrinsic transition time of the same magnitude which means larger voltage variations on the 1 ps time scale. The adiabatic approximation and $a$ fortiori the RSJC model are not any more well justified.

\section{The first order series expansion of the Josephson current.}

There are two ways to overcome the limitations of the RSJC model. The development of a better approximation or the full numerical resolution of (Eq. (1)). The latter method is more accurate with the powerful available computers. It was largely developed by Harris in the frequency domain [8-10] and then extended to the time domain [11]. Although Gayley [14] showed that some truncation could be done in numerical calculations, the major problem of this approach is to require long computer time and large memory space to simulate a single junction. This makes unconvenient their extension to logic gates and circuits.

The development of a series expansion (S.E.) of equation (1) was originally suggested by Larkin and Ovchinikov [7] but was never exploited to our knowledge. We found that the first order series expansion in time of equation (1) offers an improved model of fast junctions. It may be formulated in a way very similar to the RSJC model (Eq. (4)), thus allowing the same type of algorithm and efficient calculations.

The first order S.E. assumes a linear variation of the voltage $V\left(t^{\prime}\right)$ within a calculation time step of the junction dynamics,

$$
V\left(t^{\prime}\right)=V(t)+\left(t^{\prime}-t\right) \times \mathrm{d} V / \mathrm{d} t .
$$


Then

$$
\exp \left[i \int_{t}^{t+t_{1}} \frac{e V\left(t^{\prime}\right)}{\hbar} \mathrm{d} t^{\prime}\right] \simeq \exp \left(i \frac{e}{\hbar} V(t) \times t_{1}\right)\left[1+\frac{i}{2} \frac{e}{\hbar} \frac{\partial V}{\partial t} t_{1}^{2}\right]
$$

The approximation $\mathrm{e}^{x}=1+x$ in equation (6) gives a $5 \%$ accuracy if $x=0.3$ which means $(\mathrm{d} V / \mathrm{d} t)=6 \mathrm{mV} / \mathrm{ps}$ or a Mc Cumber parameter $\beta=2$. This indicates already that Josephson devices may be modelled reasonably with the first order S.E. if $\beta>2$. This is roughly the actual limitation of the Josephson technology in small circuits.

Using equation (6) in equation (1) the Josephson junction may be described with the equations

$$
I(t)=I_{\mathrm{c}}(V) \sin [\varphi(t)]+I_{\mathrm{pqp}}(V) \cos [\varphi(t)]+I_{\mathrm{qp}}(V)+(C+A) \times \frac{\mathrm{d} V}{\mathrm{~d} t}
$$

where

$$
A=-\frac{1}{2} \frac{\hbar}{e}\left[\frac{\partial^{2} I_{\mathrm{c}}(V)}{\partial V^{2}} \cos [\varphi(t)]+\frac{\partial^{2} I_{\mathrm{pqp}}(V)}{\partial V^{2}} \sin [\varphi(t)]+\frac{\partial^{2} I_{\mathrm{qpr}}(V)}{\partial V^{2}}\right]
$$

and

$$
\frac{\mathrm{d} \varphi}{\partial t}=-(2 e / \hbar) \times V(t)
$$

The calculation of the current components in equations (7-8) is made using BCS theory and Harris formulae [8]. Some precautions must be taken to determine the second derivatives when strong variations of the current components versus voltage occur, i.e. near $V \sim 0$ and $V \sim V_{\mathrm{g}}$. In these cases, Larkin and Ovchinikov give approximate formulae for currents (expressions (26) and (27) in [7]). Their analytical derivatives have been crosschecked with numerical calculations starting with Harris formulae [8]. However the physical rounding of the Riedel peak [17] is taken into account by limiting the Riedel peak amplitude to $3 \times I_{c}(0)$. The normalized values of the current components and their derivatives versus voltage constitute tables which are used to simulate devices or circuits. Such data are given for a niobium-lead technology in table I.

Since the second derivatives of the current components are negative in most of the $(I V)$ characteristic, the new term in equation (7) behaves as a voltage dependent capacitance $A$ with an average positive dynamic value in parallel with the junction static capacitance $C$. The physical validity of the increased effective capacitance of low $\beta$ junctions is confirmed by a recent theoretical result. A new quantum mechanical description of the phase difference in a superconducting tunnel junction [16] underlines in fast junctions the existence of a significant added term contributing to the effective capacitance (Eq. (33) of Ref. [16]). Then it may be expected with low $\beta$ junctions that the first order S.E. method gives slower time responses than the RSJC model. This is illustrated in the figures 1-4 of reference [12].

We now turn to the validity of the S.E. and remind first the assumpitions at the basis of (Eq. (1)). They are the general assumptions of the Werthamer theory related to the tunnelling Hamiltonian. The super- conductors are described by the BCS theory in the weak electron-coupling limit [9]. The theory is further limited to devices in which current densities are not too large. Then equilibrium within the electrodes is not substantially disturbed e.g. with quasi-particle injection [3].

The derivation of the S.E. method (Eq. (7)) neglects space dependent effects in the junctions. This is well justified since picosecond junctions have transverse dimensions in the micrometer range which are much smaller than the electromagnetic dimensions of interest.

We have performed an evaluation of the second order terms in the series expansion of $V(t)$. The $\left(\partial^{2} V / \partial t^{2}\right)$ derivative terms bring the following contribution to be added in equation (7),

$$
\begin{aligned}
\frac{\partial^{2} V}{\partial t^{2}} \times \frac{1}{6} \times \frac{e}{\hbar} \times & {\left[\frac{\partial^{3} I_{c}(V)}{\partial V^{3}} \sin [\varphi(t)]-\right.} \\
& \left.-\frac{\partial^{3} I_{\mathrm{pqp}}(V)}{\partial V^{3}} \cos [\varphi(t)]-\frac{\partial^{3} I_{\mathrm{qp}}}{\partial V^{3}}\right]
\end{aligned}
$$

The third derivatives of the current components in equation (10) are also tabulated in table I for the niobium-lead technology.

The numerical calculation of equation (10) shows that its contribution relatively to the $A$ term (Eq. (8)) is negligible in junctions with $\beta>5$. In junctions with $\beta<2$, the successive terms of the series expansion cannot be any more considered as correcting terms.

The table II summarizes the range of validity of the approximations in the series expansions of the equations (5-6) leading to equation (8). It also gives its range of interest relatively to the RSJC model as it will be illustrated in section 4 . 
Table I. - Josephson current components and their voltage derivatives, calculated with Harris formulae [8] for $T / T_{\mathrm{c}}=0.5$ and using numerical methods for the derivatives, the currents and voltages are given in relative units of the critical current $I_{\mathrm{c}}(0)$ and the gap voltage $V_{\mathrm{g}}$.

\begin{tabular}{|c|c|c|c|c|c|c|c|c|c|}
\hline$V$ & $I_{\mathrm{c}}$ & $I_{\mathrm{qp}}$ & $I_{\mathrm{pqp}}$ & $\partial^{2} I_{\mathrm{c}} / \partial V^{2}$ & $\partial^{2} I_{\mathrm{pqp}} / \partial V^{2}$ & $\partial^{2} I_{\mathrm{qpr}} / \partial V^{2}$ & $\partial^{3} I_{\mathrm{c}} / \partial V^{3}$ & $\partial^{3} I_{\mathrm{pqp}} / \partial V^{3}$ & $\partial^{3} I_{\mathrm{qp}} / \partial V^{3}$ \\
\hline 0 & 1 & 0 & 0 & -15 & -3 & -19 & 2000 & 610 & 630 \\
\hline 0.057 & 1.01 & 0.02 & 0.01 & -1 & -2 & -3 & 35 & 50 & 50 \\
\hline 0.28 & 1.06 & 0.04 & 0.02 & 0.4 & -0.1 & -2 & 2 & 1 & 2 \\
\hline 0.56 & 1.16 & 0.04 & 0.02 & 1.6 & 0 & -4 & 8 & -2 & 0.2 \\
\hline 0.89 & 1.52 & 0.03 & 0.01 & 28 & 0 & -34 & 480 & -0.1 & 0 \\
\hline 0.94 & 1.7 & 0.04 & 0.01 & 99 & 0 & -109 & 2950 & -0.1 & 0 \\
\hline 0.97 & 1.92 & 0.04 & 0.01 & 350 & 0 & -350 & $24 \times 10^{3}$ & -0.1 & 0 \\
\hline 0.98 & 2.1 & 0.04 & 0.01 & 800 & 0 & -800 & $8 \times 10^{4}$ & -0.1 & 0 \\
\hline 0.988 & 2.2 & 0.04 & 0.01 & 2200 & 0 & -2200 & $37 \times 10^{4}$ & -0.1 & 0 \\
\hline 1 & 3 & 0.04 & 0.01 & $10^{4}$ & 0 & $-10^{4}$ & $10^{6}$ & -0.1 & 0 \\
\hline 1 & 3 & 1 & -1 & $10^{4}$ & 50 & $-10^{4}$ & $-10^{6}$ & $-10^{4}$ & $10^{4}$ \\
\hline 1.012 & 2.2 & 1.04 & -1 & 2200 & 40 & -2200 & $-37 \times 10^{4}$ & -9600 & 9400 \\
\hline 1.02 & 2 & 1.06 & -1 & 800 & 7 & -800 & $-8 \times 10^{4}$ & -820 & 777 \\
\hline 1.03 & 1.9 & 1.07 & -1 & 350 & 2.5 & -350 & $-24 \times 10^{3}$ & -180 & 175 \\
\hline 1.06 & 1.6 & 1.12 & -0.98 & 90 & 0.95 & -90 & -2950 & -26 & 18 \\
\hline 1.1 & 1.5 & 1.2 & -0.97 & 30 & 0.4 & -30 & -723 & -6 & 4 \\
\hline 1.2 & 1.2 & 1.4 & -0.94 & 9.5 & 0.05 & -10 & -90 & -2 & 2 \\
\hline 1.4 & 0.9 & 1.7 & -0.88 & 2.6 & -0.2 & -3 & -12 & -0.4 & 0.8 \\
\hline 3 & 0.4 & 4 & -0.58 & 0.1 & -0.05 & -0.1 & -0.1 & -0.4 & 0.03 \\
\hline 4 & 0.3 & 5.4 & -0.49 & 0.1 & -0.05 & -0.1 & -0.1 & -0.4 & 0.03 \\
\hline
\end{tabular}

Table II. - Range of validity of the approximations leading to equation (8).

$$
\begin{array}{lllll}
\beta & 50 & 20 & 5 & 2
\end{array}
$$

Validity very good accuracy better Not valid of Eq. (8) $\quad$ than $10 \%$

Deviation small significant
to RSJC

There are only two examples available in the literature of the temporal response of switching junctions simulated using the direct numerical resolution of the Werthamer equations $[11,14]$. We have performed the simulation of a junction with $\beta=5$ in the same circuit conditions as Harris ([11], Fig. 2, p. 5189). There is no measurable difference, which is another confirmation of the validity of our approach. The simulation performed by Gayley [14] corresponds to a junction with $\beta<0.5$ which is far off the range of validity of the S.E. model.

Another point is the amplitude of the Josephson voltage oscillation for picosecond current biased junctions in the resistive state at $V=V_{\mathrm{g}}$. Voltage oscillations related to the Josephson $\mathrm{AC}$ current occur depending on the junction load. The junction may oscillate strongly across the gap voltage at a frequency of the order of the gap frequency $[11,12]$. Since large variations of voltage occur on the corresponding time scale, the first order S.E. overestimates the physical process and may not give accurate results.

The AC voltage amplitude may be calculated directly in stationnary conditions when $V \sim V_{g}$ and $I_{\mathbf{T}}=$ constant as shown in the Appendix. These 
equations allow a proper evaluation of the alternating voltage across a current biased Josephson junction. The calculations may be extended to higher harmonics which have much smaller amplitudes than $V(2 \omega)$ at large dc voltage $\left(>V_{g}\right)$. A comparison of the results with the S.E. (first order) calculations indicates that around the gap $\left(V_{\mathrm{g}} \pm 10 \%\right)$ the adiabatic approximation (zero order) gives a better precision $( \pm 10 \%)$

\section{Switching characteristics of loaded picosecond Josephson junctions.}

The dynamic behaviour of Josephson junctions depends strongly on their loading conditions. The switching waveform variations between the S.E. model and the RSJC model have been illustrated in a previous publication [12]. We present in this section a systematic comparison of the characteristic switching times of the circuit in figure 1a. A single junction defined by the parameters $I_{0}, R_{\mathrm{N}}, C, \beta$ is loaded with $R_{\mathrm{L}}$. It is driven with a bias current $I_{\mathrm{g}}<I_{0}$ and the control current is $I_{\mathrm{c}}$. The following parameter variations are considered. The Mc Cumber parameter $\beta$ ranges from 2 to 50 . If $\beta=50$ the RSJC and S.E. models give quite identical results. If $2 \lesssim \beta \lesssim 5$ the S.E. model approaches its limit and the results must be considered more qualitatively. The different loading conditions $\left(R_{\mathrm{L}} / R_{\mathrm{N}}\right)=100,1,0.5,0.2$ are considered. $\left(R_{\mathrm{L}} / R_{\mathrm{N}}\right)=100$ pictures an unloaded junction, $\left(R_{\mathrm{L}} / R_{\mathrm{N}}\right)=1$ refers to a standard output load of a direct coupled logic (DCL) or RCJL gate (labelled $A$ in the following). $\left(R_{\mathrm{L}} / R_{\mathrm{N}}\right)=0.2$ is a load condition often used in the literature for the input junction of RCJL gates (labelled B). We have considered two junction bias conditions : i) $I_{\mathrm{g}}=0.9 I_{0}, I_{\mathrm{c}}=0.2 I_{0}$ corresponding to a small overdrive (labelled C), ii) $I_{\mathrm{g}}=0.8 I_{0}, I_{\mathrm{c}}=0.4 I_{0}$ representative of average driving conditions of the input junction of a DCL or RCJL gate (labelled D). The time scale of the control current ramp $I_{c}$ (Fig. 1b-c) fits in each case the junction intrinsic speed characterized with the $R_{\mathrm{N}} C$ product. Figures $1 \mathrm{~b}$ and $1 \mathrm{c}$ also define the main characteristic times of interest in the switching process, the turn-on-delay $t_{\text {od }}$ and the total switching time $t_{\mathrm{t}}$. The amplitude of the Josephson oscillation is small (Fig. 1b) when the junction is lightly loaded, then $t_{\text {od }}$ and $t_{\mathrm{t}}$ may be defined accurately. On the other hand figure $1 \mathrm{c}$ indicates that in strongly loaded junctions, the dc bias point is under the gap voltage, strong Josephson voltage oscillations occur and the definition of $t_{\mathrm{od}}$ and $t_{\mathrm{t}}$ is less accurate.

The comparison of the RSJC and S.E. models is illustrated with plots of the ratios of the characteristic times of both models $\left(t_{\mathrm{SE}} / t_{\mathrm{RSIC}}\right)$. This is done both for the total switching time $t_{\mathrm{t}}$ and the turn on delay $t_{\mathrm{od}}$ as a function of the Mc Cumber parameter $\beta$. The junction loading condition $\left(R_{\mathrm{L}} / R_{\mathrm{N}}\right)$ is taken as a parameter. Figure 2a-b corresponds to the small overdrive condition (C) and figure $3 a-b$ shows the

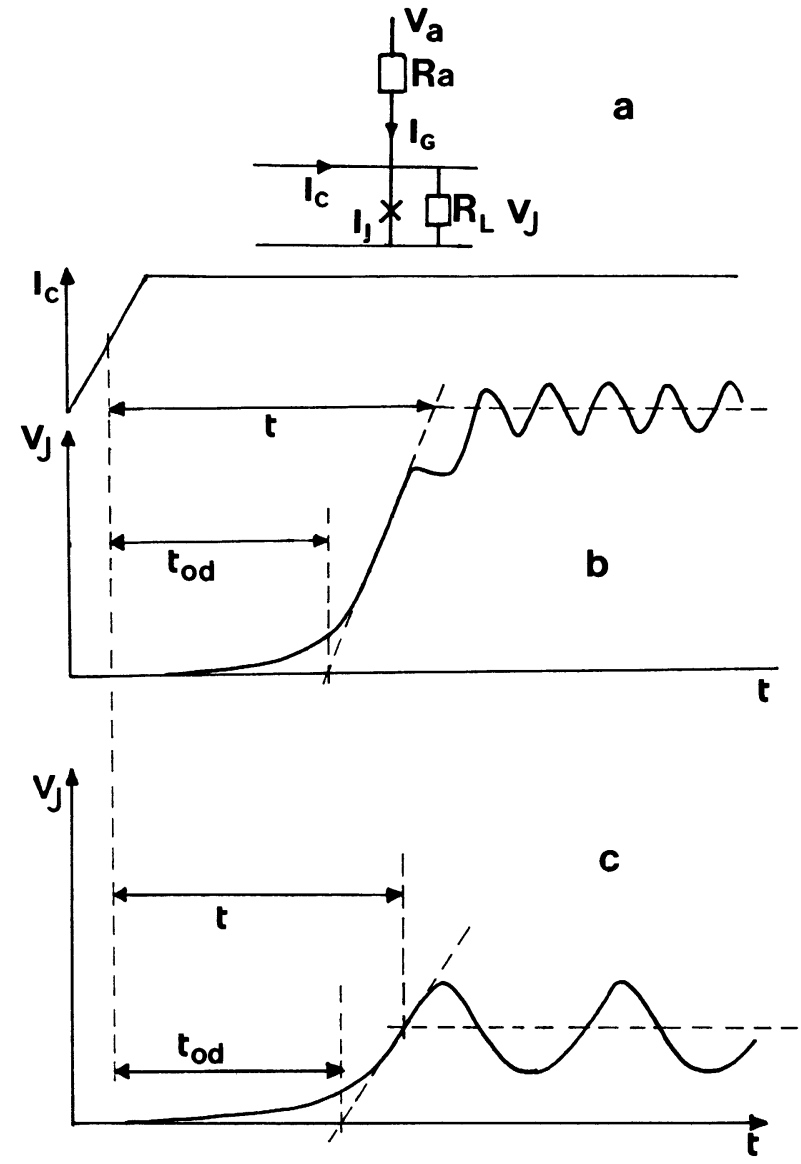

Fig. 1. - Evaluation of fast Josephson junction switching times. Figure 1a represents the simulated circuit with the junction parameters $\left(I_{0}, \beta, R_{\mathrm{N}}\right)$. The turn-on-delay and the Josephson oscillation at the gap distort the voltage variation in fast junctions from the usual trapezoidal switching waveform (see Ref. [11]). Figures $1 \mathrm{~b}$ and $1 \mathrm{c}$ show the definitions of the turn on delay $t_{\mathrm{od}}$ and the total switching time $t_{\mathrm{t}}$ in lightly loaded junctions $\left(R_{l} / R_{\mathrm{N}} \ll 1\right)$ and strongly loaded junctions $\left(R_{l} / R_{\mathrm{N}}<1\right)$.

average drive condition (D). Absolute switching time and $t_{\mathrm{od}}$ are summarized in table III.

The figures emphasize the following points :

- The RSJC and S.E. models give very similar results with $\beta \gtrsim 15$ junctions.

- On the other side the S.E. model is more precise with lightly loaded picosecond junctions. The differences in switching time $t_{\mathrm{t}}$ and $t_{\mathrm{od}}$ given by the two models range between 30 and $70 \%$ when $\beta_{\mathrm{c}}=2$.

- The effect of the successive terms of the S.E. model is damped in strongly loaded junctions $\left(R_{\mathrm{L}} / R_{\mathrm{N}} \lesssim\right.$ $0.5)$ and the RSJC model remains satisfactory within $10 \%$.

Finally the figure 4 illustrates the relative improvement of junction speed which is obtained with junctions of small $\beta$ (or $R_{\mathrm{N}} C$ ). It shows the reduction of the total switching time $t_{\mathrm{SE}}$ given by the S.E. model as a function of $\beta ; t_{\mathrm{SE}}$ is measured in units of the total switching time $t_{\mathrm{RSJC}}$ given by the RSJC model at 

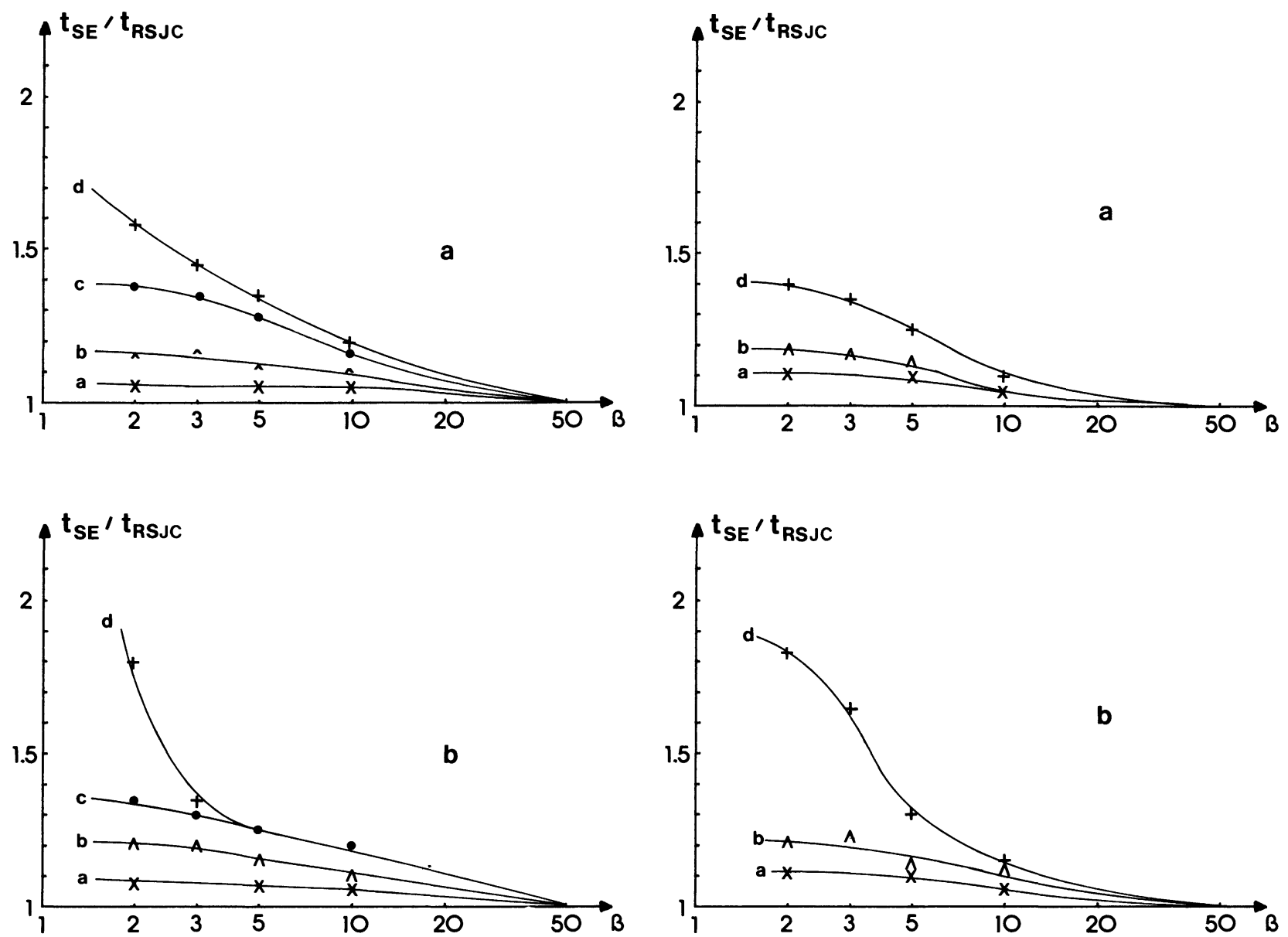

Fig. 2. - Comparison of the S.E. and RSJC models. Figure 2a is related to the total junction switching time $t_{\mathrm{t}}$ and figure $2 \mathrm{~b}$ to the junction turn-on-delay $t_{\mathrm{od}}$. The variations $t_{\mathrm{SE}} / t_{\mathrm{RSJC}}$ are given versus the Mc Cumber parameter, in different loading conditions $\left(R_{\mathrm{L}} / R_{\mathrm{N}}\right):$ a) 0.2 , b) 0.5 , c) 1 , d) 100 . The junction is in small overdrive conditions : $I_{\mathrm{g}}=0.9 I_{0}$, $I_{\mathrm{c}}=0.2 I_{0}$.

Fig. 3. - Comparison of the S.E. and RSJC models. The conditions are the same as in figure 2 but the junction is in average overdrive conditions : $I_{\mathrm{g}}=0.8 I_{0}, I_{\mathrm{c}}=0.4 I_{0}$.

Table III. - Total switching time and turn on delay for different $\beta$ values and loading condition. The junction is in average conditions as in figure 3.

\begin{tabular}{rccccc}
$\beta$ & $T_{\mathrm{T}}(\mathrm{S}$.E. $)$ & $T_{\mathrm{T}}(\mathrm{RSJC})$ & $T_{\text {OD }}($ S.E. $)$ & $T_{\text {OD }}($ RSJC $)$ & $R_{\mathrm{L}} / R_{\mathrm{N}}$ \\
\hline 50 & 16 & 16 & 4 & 4 & \\
10 & 5 & 4.5 & 1.7 & 1.5 & 10 \\
5 & 3.1 & 2.5 & 1.5 & 1.15 & \\
2 & 2.25 & 1.6 & 1.1 & 0.6 & \\
\hline 50 & 13 & 13 & 5.5 & 5.5 & 0.5 \\
10 & 4.6 & 4.3 & 3.5 & 3 & \\
5 & 3.9 & 3.4 & 2.75 & 2.5 & \\
2 & 3.3 & 2.75 & 2.40 & 2 & 0.2 \\
\hline 50 & 11 & 11 & 7.5 & 7.5 & \\
10 & 7 & 6.6 & 5.7 & 5.4 & \\
5 & 6.5 & 5.9 & 5.0 & 4.5 & \\
2 & 6 & 5.5 & 4.75 & 4.25 &
\end{tabular}




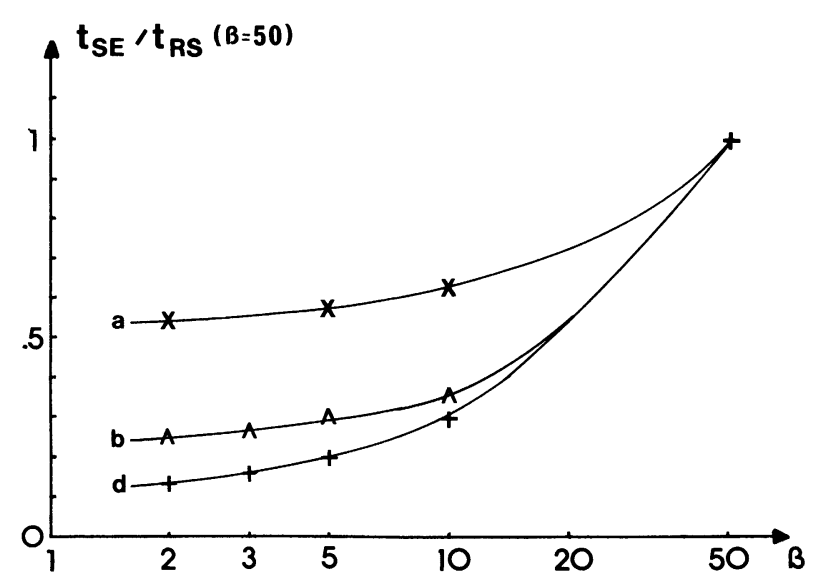

Fig. 4. - Reduction of the total switching time $t_{\mathrm{SE}}$ as a function of $\beta ; t_{\mathrm{SE}}$ is measured in units of $t_{\mathrm{RSJC}}(\beta=50)$.

$\beta=50\left(t_{\mathrm{SE}} \simeq t_{\mathrm{RSJC}}\right.$ at $\left.\beta=50\right)$. The figure clearly shows that the total switching time is reduced strongly with lightly loaded junctions (curves $b$ and $d$ ) when the Mc Cumber parameter $\beta_{\mathrm{c}}$ decreases from 50 to 5 . The speed improvement is far less important with strongly loaded junctions (curve a). A further reduction of $\beta(\beta<5)$ gives additionnal speed reduction only for unloaded junctions (curve $d$ ). In all cases, the total switching time decreases only very slowly if $\beta_{c} \leqslant 2$. This means that extremely fast junctions
( $\sim 1 \mathrm{ps})$ are worth only in special circuits but give no speed benefit in digital circuits. Careful circuit design limiting junction loading is also strongly recommanded with any picosecond Josephson technology [15].

\section{Conclusion.}

The S.E. method allows an efficient and precise modeling of picosecond $(\beta \gtrsim 2)$ Josephson junctions and circuits. It introduces an equivalent time dependent and non linear capacitance. This reduces the junction speed relatively to the RSJC model. The different contributions to the term describing this capacitance are tabulated so that the increase in CPU time relatively to the RSJC model is negligible. The method has been applied to the design of a logic gate family and a Josephson adder is under study [15]. The switching dynamics of a loaded junction analysed with both models shows that significant errors are introduced by the RSJC model with lightly loaded $\left(R_{\mathrm{L}} / R_{\mathrm{N}} \gtrsim 1\right)$ picosecond $(\beta \lesssim 10)$ junctions. On the other side the RSJC model remains satisfactory with strongly loaded junctions. This study also emphasizes that down scaling of Josephson logic circuits is not straightforward. The intrinsic speed of picosecond junctions may only be transferred at the scale of a circuit if appropriate design based on lightly loaded junctions is performed [15].

\section{Appendix}

AmPlitude of THE voltage oscillations AT tHE GAP voltage. - We use equation (16) of reference [6] with the sign conventions of Harris [7].

$$
V(t)=V_{\mathrm{s}}+\frac{1}{2 \pi} \int V(\omega) \mathrm{e}^{-i \omega t} \mathrm{~d} \omega
$$

if

$$
\begin{aligned}
& I(t)=\operatorname{Jm}\left[\frac{e V(\omega)}{2 \pi \hbar \omega} \int \mathrm{d} \omega \mathrm{e}^{-i \omega t}\right. {\left[\left(I_{\mathrm{qp}}\left(\frac{\hbar \omega}{e}+V_{\mathrm{s}}\right)-I_{\mathrm{qp}}\left(V_{\mathrm{s}}\right)\right)+I_{\mathrm{qpr}}\left(\frac{\hbar \omega}{e}+V_{\mathrm{s}}\right)-I_{\mathrm{qpr}}\left(V_{\mathrm{s}}\right)\right]+\int \mathrm{d} \omega \times } \\
&\left.\times \mathrm{e}^{i(\varphi-\omega t)} \times\left[I_{\mathrm{c}}\left(\frac{\hbar \omega}{e}+V_{\mathrm{s}}\right)-I_{\mathrm{c}}\left(V_{\mathrm{s}}\right)+i\left(I_{\mathrm{pqp}}\left(\frac{\hbar \omega}{e}+V_{\mathrm{s}}\right)-I_{\mathrm{pqp}}\left(V_{\mathrm{s}}\right)\right)\right]\right]+I_{\mathrm{dc}}
\end{aligned}
$$

$V_{\mathrm{s}}$ is the average dc voltage and $V(\omega)$ are the harmonics of the alternative voltage component across the device, $I_{\mathrm{dc}}$ is the dc component of the device current. The above expression is valid if harmonic amplitudes are not too large, $V(\omega)<V_{\mathrm{s}}$. That the device is biased at constant current, the alternative current components must be zero. This gives conditions for the calculation of the voltage harmonic amplitudes. We define respectively the fundamental and first harmonic voltage amplitudes as

$$
V_{1}(\omega)+i V_{2}(\omega) \quad \text { and } \quad V_{1}^{\prime}(2 \omega)+i V_{2}^{\prime}(2 \omega)
$$

We also introduce the current components

$$
\begin{array}{ll}
\left(I_{1}\right)=I_{\mathrm{c}}\left(3 V_{\mathrm{s}}\right)-I_{\mathrm{c}}\left(V_{\mathrm{s}}\right) & \left(I_{1}^{\prime}\right)=I_{\mathrm{c}}\left(5 V_{\mathrm{s}}\right)-I_{\mathrm{c}}\left(V_{\mathrm{s}}\right) \\
\left(I_{2}\right)=I_{\mathrm{pqp}}\left(3 V_{\mathrm{s}}\right)-I_{\mathrm{pqp}}\left(V_{\mathrm{s}}\right) & \left(I_{3}^{\prime}\right)=I_{\mathrm{qp}}\left(5 V_{\mathrm{s}}\right)-I_{\mathrm{qp}}\left(V_{\mathrm{s}}\right) \\
\left(I_{3}\right)=I_{\mathrm{qp}}\left(3 V_{\mathrm{s}}\right)-I_{\mathrm{qp}}\left(V_{\mathrm{s}}\right) & \left(I_{4}^{\prime}\right)=I_{\mathrm{qpr}}\left(5 V_{\mathrm{s}}\right)-I_{\mathrm{qpr}}\left(V_{\mathrm{s}}\right) \\
\left(I_{4}\right)=I_{\mathrm{qpr}}\left(3 V_{\mathrm{s}}\right)-I_{\mathrm{qpr}}\left(V_{\mathrm{s}}\right) . &
\end{array}
$$


We obtain the voltage amplitudes

with

$$
\begin{aligned}
V_{1}(\omega) & =-\frac{\left(I_{3}\right) \times I_{\mathrm{pqp}}\left(V_{\mathrm{s}}\right)+I_{\mathrm{c}}\left(V_{\mathrm{s}}\right)\left(2 \times C \omega_{\mathrm{s}} V_{\mathrm{s}}+\left(I_{4}\right)\right)}{\left(I_{3}\right)^{2}+\left(2 C \omega_{\mathrm{s}} V_{\mathrm{s}}+\left(I_{4}\right)\right)^{2}} \times 4 \pi V_{\mathrm{s}} \\
V_{2}(\omega) & =-\frac{-\left(I_{3}\right) \times I_{\mathrm{c}}\left(V_{\mathrm{s}}\right)+I_{\mathrm{pqp}}\left[2 C \omega_{\mathrm{s}} V_{\mathrm{s}}+\left(I_{4}\right)\right]}{\left(I_{3}\right)^{2}+\left(2 C \omega_{\mathrm{s}} V_{\mathrm{s}}+\left(I_{4}\right)\right)^{2}} \times 4 \pi V_{\mathrm{s}} \\
V_{1}^{\prime}(2 \omega) & =\frac{\left(I_{3}^{\prime}\right) a^{\prime}+b^{\prime}\left(2 C \omega_{\mathrm{s}} V_{\mathrm{s}}+\left(I_{4}^{\prime}\right)\right)}{\left(I_{3}^{\prime}\right)^{2}+\left(2 C \omega_{\mathrm{s}} V_{\mathrm{s}}+\left(I_{4}^{\prime}\right)\right)^{2}} \\
V_{2}^{\prime}(2 \omega) & =\frac{-\left(I_{3}^{\prime}\right) b^{\prime}+a^{\prime}\left(2 C \omega_{\mathrm{s}} V_{\mathrm{s}}+\left(I_{4}^{\prime}\right)\right)}{\left(I_{3}^{\prime}\right)^{2}+\left(2 C \omega_{\mathrm{s}} V_{\mathrm{s}}+\left(I_{4}^{\prime}\right)\right)^{2}}
\end{aligned}
$$

$$
\begin{aligned}
& a^{\prime}=-2\left[I_{\mathrm{c}}\left(3 V_{\mathrm{s}}\right) V_{2}(\omega)+I_{\mathrm{pqp}}\left(3 V_{\mathrm{s}}\right) V_{1}(\omega)\right] \\
& b^{\prime}=-2\left[I_{\mathrm{c}}\left(3 V_{\mathrm{s}}\right) V_{1}(\omega)-I_{\mathrm{pqp}}\left(3 V_{\mathrm{s}}\right) V_{2}(\omega)\right] .
\end{aligned}
$$

\section{References}

[1] MC Cumber, D. E., J. Appl. Phys. 39 (1968) 3113.

[2] Stewart, W. C., Appl. Phys. Lett. 12 (1968) 277.

[3] Mc Donald, D. G., Peterson, R. L., Hamilton, C. A., HARRIS, R. E., KAUTZ, R. L., IEEE Trans Electron Devices ED 27 (1980) 1945.

[4] Wolf P., Van Zeghbroeck, B. J., Deutsch, U., IEEE Trans. Magnetics MAG 21 (1985) 226.

[5] Sone, J., Tsai, J., ABe, H., IEEE J. Solid State Circuits SC 20 (1985) 1056.

[6] Werthamer, N. R., Phys. Rev. 147 (1966) 255.

[7] Larkin, A. I., Ovchinikov, Yu. N., Soviet. Phys. JETP 24 (1967) 1035.

[8] Harris, R. E., Phys. Rev. 10 (1974) 84.

[9] Harris, R. E., Phys. Rev. 11 (1975) 3329.
[10] Harris, R. E., Phys. Rev. 13 (1976) 3818.

[11] Harris, R. E., J. Appl. Phys. 48 (1977) 5188.

[12] De Lustrac, A., Crozat, P., Adde, R., IEEE Trans. Magn. MAG 19 (1983) 1221.

[13] Kulik, I. O., Yanson, I. K., The Josephson effect in Superconductive tunneling structures, ch. 1.3 (Keter Press, Jerusalem) 1972.

[14] Gayley, R. I., J. Appl. Phys. 52 (1981) 1411.

[15] De Lustrac, A., Crozat, P., Adde, R., ICSQUid Conference, Berlin, June 1985.

[16] Eckern, U., Schon, G., Ambegaokar, V., Phys. Rev. B 30 (1984) 6419.

[17] Vernet, G., Henaux, J.-C., Adde, R., Appl. Phys. Lett. 55 (1984) 3020. 